\title{
Partial orientation pop-out helps difficult search for orientation
}

\author{
ELIZABETH S. OLDS \\ Wilfrid Laurier University, Waterloo, Ontario, Canada \\ and \\ WILLIAM B. COWAN and PIERRE JOLICCEUR \\ University of Waterloo, Waterloo, Ontario, Canada
}

\begin{abstract}
We interrupted pop-out search before it produced a detection response by adding extra distractors to the search display. We show that when pop-out for an orientation target fails because of this interruption, it nevertheless provides useful information to the processes responsible for difficult search. That is, partial pop-out assists difficult search. This interaction has also been found for color stimuli (Olds, Cowan, \& Jolicour, 2000a, 2000b). These results indicate that interactions and/or overlap between the mechanisms responsible for pop-out and the mechanisms responsible for difficult search may be quite general in early visual selection.
\end{abstract}

Vision provides people with an enormous amount of information. Because it is impossible to process all of this information in detail, limited subsets must be quickly selected for further analysis. How is this selection performed? The mechanisms responsible for visual selection can be elucidated by data from search tasks. In a search task, observers determine whether or not a target appears among distractors (other items) in a complex display. Researchers classify search performance in terms of response time (RT) patterns. In pop-out search, RTs are fast, and RT for correct responses is virtually independent of the number of items in the display. In difficult search, RTs are much longer and generally increase linearly with the number of items (Treisman \& Gelade, 1980). Pop-out search is possible for some target-distractor relationships but not others. Many researchers believe that early "preattentive" mechanisms in the brain perform pop-out search in parallel across the entire display; difficult search occurs when these mechanisms cannot detect the target and "attention" is required (Treisman \& Gelade, 1980; Wolfe, 1994). Most work on attention implicitly assumes that preattentive processing is all-or-nothing (Treisman \& Gelade, 1980; Wolfe, 1994), despite suggestions that processing could develop gradually (e.g., Wolfe, 1994, p. 229; Wolfe, Cave, \& Franzel, 1989, p. 428).

Olds, Cowan, and Jolicœur (2000a, 2000b) have shown, however, that preattentive processing of color is not allor-nothing but can, in fact, be dissected both temporally and spatially. They interrupted pop-out search at interme-

This research was funded by a Human Frontier Science Program grant awarded to W.B.C. and P.J. Correspondence should be addressed to E. S. Olds, Department of Psychology, Wilfrid Laurier University, 75 University Avenue West, Waterloo, ON, N2L 3C5 Canada (e-mail: eolds@,wlu.ca). diate points in processing, and the results showed that even when pop-out search fails, its partially completed computations can be used to assist other, slower search processes. The results of these experiments provide basic constraints that must be satisfied by any model of early vision and visual selection. Because all visual processing is affected by which portions of the visual field these early mechanisms select for further analysis, it is important to determine the nature of each selection mechanism, as well as interactions between the mechanisms.

All of the results of Olds et al. $(2000 \mathrm{a}, 2000 \mathrm{~b})$ were based on experiments in the color domain. Are the results specific to color, or can they be generalized to other stimulus dimensions? In this paper, we investigate interactions between pop-out and difficult search in the orientation domain. The goal is to discover whether the mechanisms involved in search facilitation are general. If we can demonstrate this facilitation in a large number of different stimulus domains, and these mechanisms operate in similar ways in many different cases, search facilitation could provide the basis for a universal principle in visual selection. On the other hand, we could find that search facilitation operates only in the color domain, and not, for example, in the orientation domain. This would be evidence that the search facilitation we previously found (Olds et al., 2000a, 2000b) for color stimuli occurs relatively early in processing (i.e., at the level of some feature maps, in Treisman and Gelade's, 1980, terms) rather than at a later stage (i.e., at the level of feature conjunctions, see, e.g., Wolfe, 1994).

In the experiments of Olds et al. (2000a, 2000b), an observer reported the presence or absence of a target, seen among distractors. The target $(T)$, present on half the trials, was a disk of a particular color; the distractors were disks of different colors. Each trial began with a central "+," which the observer was asked to fixate $(400 \mathrm{msec})$, 
followed by a blank interval $(400 \mathrm{msec})$ and then the stimulus. The first portion of a trial presented the target along with distractors of one color (D1). Pop-out search is possible for such stimuli. After a delay (stimulus onset asynchrony, or SOA), distractors of a second color (D2) were added (in the locations not already occupied by D1 distractors). The stimulus colors are defined in a 3dimensional color space (Wyszecki \& Stiles, 1982) with one dimension specifying luminance and two dimensions specifying chromaticity; the stimuli all had the same luminance so they varied only in 2-dimensional chromaticity. D2 was chosen so that $\mathrm{T}$ was between D1 and D2 in color space, making a display for which popout search was not possible ( $\mathrm{T}$ is "not linearly separable" from D1 and D2; see Bauer, Jolicœur, \& Cowan, 1996, 1999; D'Zmura, 1991). Thus the presence of the second set of distractors prevented processing that could lead to further pop-out. However, because the D2 distractors prevented pop-out processing without masking the existing display, difficult search could proceed after they were added. Therefore, in addition to interrupting pop-out search, these distractors also acted as a probe to examine the influence of incomplete (interrupted) pop-out search on difficult search (this is the interaction between the mechanisms responsible for these two kinds of visual selection).' Olds et al. (2000a, 2000b) analyzed the RT distributions, for conditions with different delays for the onset of the second set of distractors, to measure this interference; the technique is described in the Results section of this paper.

In the experiment described in this paper, we presented a target (on $50 \%$ of trials) among one kind of distractor (Figure 1). The target $(\mathrm{T})$ was a dark gray line oriented at $45^{\circ}$, presented on a lighter gray background. The initial distractors (D1) were lines of the same brightness, oriented at $59^{\circ}$. Search is easy in this kind of display. After a variable delay (SOA), more distractors were added to the display. These new distractors (D2) were lines oriented at $31^{\circ}$. Once the D2 distractors appeared, search was difficult. Search for T among D1 and D2 distractors is difficult because the target is between the two distractors, in the relevant feature space (which in this case is orientation); $45^{\circ}$ is between $31^{\circ}$ and $59^{\circ}$ (Wolfe, FriedmanHill, Stewart, \& O'Connell, 1992).

On some trials, pop-out was interrupted before it produced a detection response (i.e., when the D2s appeared after a brief delay). Thus the experiment allowed us to examine whether interrupted (incomplete) orientation pop-out produces intermediate computations that can be used to guide difficult search for orientation.

\section{METHOD}

\section{Equipment}

Stimuli were presented using a Macintosh PowerMac 6100 computer, MATLAB software, and Brainard's (1997) Psychophysics Toolbox routines. The coordinates of the colors were set using a Minolta Chroma Meter CS-100 and a technique described by Olds, Cowan, and Jolicœur (1999).

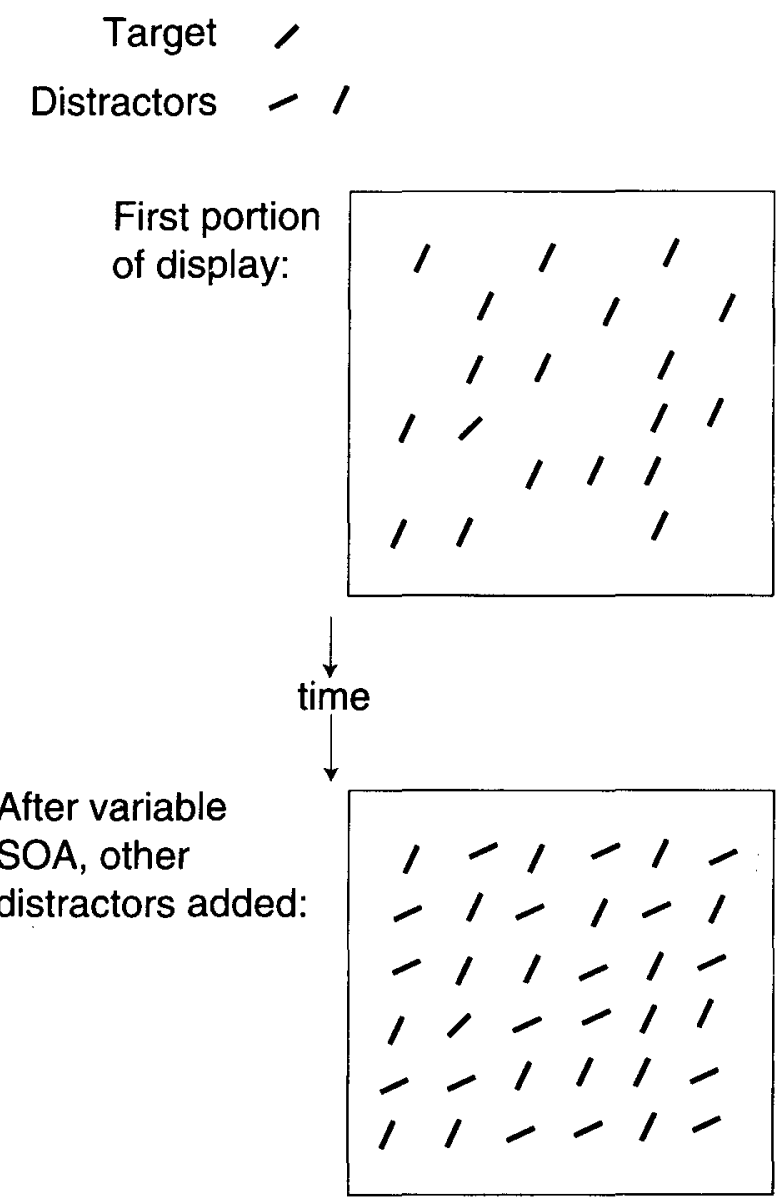

Figure 1. An illustration of the stimuli used in the experiment (top panel, first portion of the display; bottom panel, second portion of the display). Note-For illustration purposes, the distractors in the figure are rotated $20^{\circ}$ away from the target, although in the experiment the distractors were rotated $14^{\circ}$ away from the target.

\section{Observers}

Observers C.P. (female, 26 years old), L.S. (female, 23 years old), and M.T. (male, 25 years old) were paid for participation.

\section{Stimuli and Design}

Stimulus displays consisted of 36 oriented dark gray lines, each subtending approximately $0.7^{\circ}$ visual angle in length, on a lighter gray background. The lines were arranged in a $6 \times 6$ grid in the center of the screen, which subtended approximately $7^{\circ}$ horizontally and vertically. The $6 \times 6$ grid of possible locations was randomly perturbed on every trial so that each line location moved up to one fifth of a line length in the horizontal and vertical directions.

The luminance of the lines was $10 \mathrm{~cd} / \mathrm{m}^{2}$; the luminance of the background was $25 \mathrm{~cd} / \mathrm{m}^{2}$. Both had chromaticity coordinates $(.327, .332)$; the CIELuv coordinates of the lines and background were $(70, .206, .472)$ and $(100, .206, .472)$, respectively.

\section{Procedure}

Each trial began with a white fixation stimulus, presented for $400 \mathrm{msec}$. This fixation was a "+" for the first trial, and on subsequent trials following correct performance. Following an error, the fixation was a "-.". After the fixation, the screen went blank for $400 \mathrm{msec}$, and then the stimulus was presented until the observer re- 
sponded, pressing one key to indicate that the target was present and another key to indicate that it was absent. Observers were instructed to respond as quickly as possible while maintaining high accuracy.

The SOAs were $0,17,33,50,67,83,100$, and $1,000 \mathrm{msec}$, along with a control condition, SOA $=\infty$, in which the D2 distractors never appeared. The SOA $=0$ condition was a control condition in which both D1 and D2 distractors were presented from the beginning of the trial, without any delay for the onset of the D2s. Trials with different SOAs were presented intermixed. In each experimental session, the observer adapted for several minutes to the dimly illuminated room and then completed a block of trials. Each block of trials consisted of 576 experimental trials preceded by 20 practice trials. Observers L.S. and M.T. completed four sessions for a total of 2,304 experimental trials each; Observer C.P. completed eight sessions for a total of 4,608 experimental trials. ${ }^{2}$
Each observer participated in several practice sessions before the experimental sessions, practicing only the $\mathrm{SOA}=0$ and $\mathrm{SOA}=\infty$ conditions until they achieved reasonable accuracy.

\section{RESULTS}

A recursive outlier screening procedure, described in Van Selst and Jolicœur (1994), eliminated 2.4\%, 3.4\%, and $3.9 \%$ of correct trials based on their RTs for Observers C.P., L.S., and M.T., respectively. Analyses are reported for the data with the outliers removed.

Figure 2 shows mean RT and error rates for the different SOA conditions. We focus on target-present trials
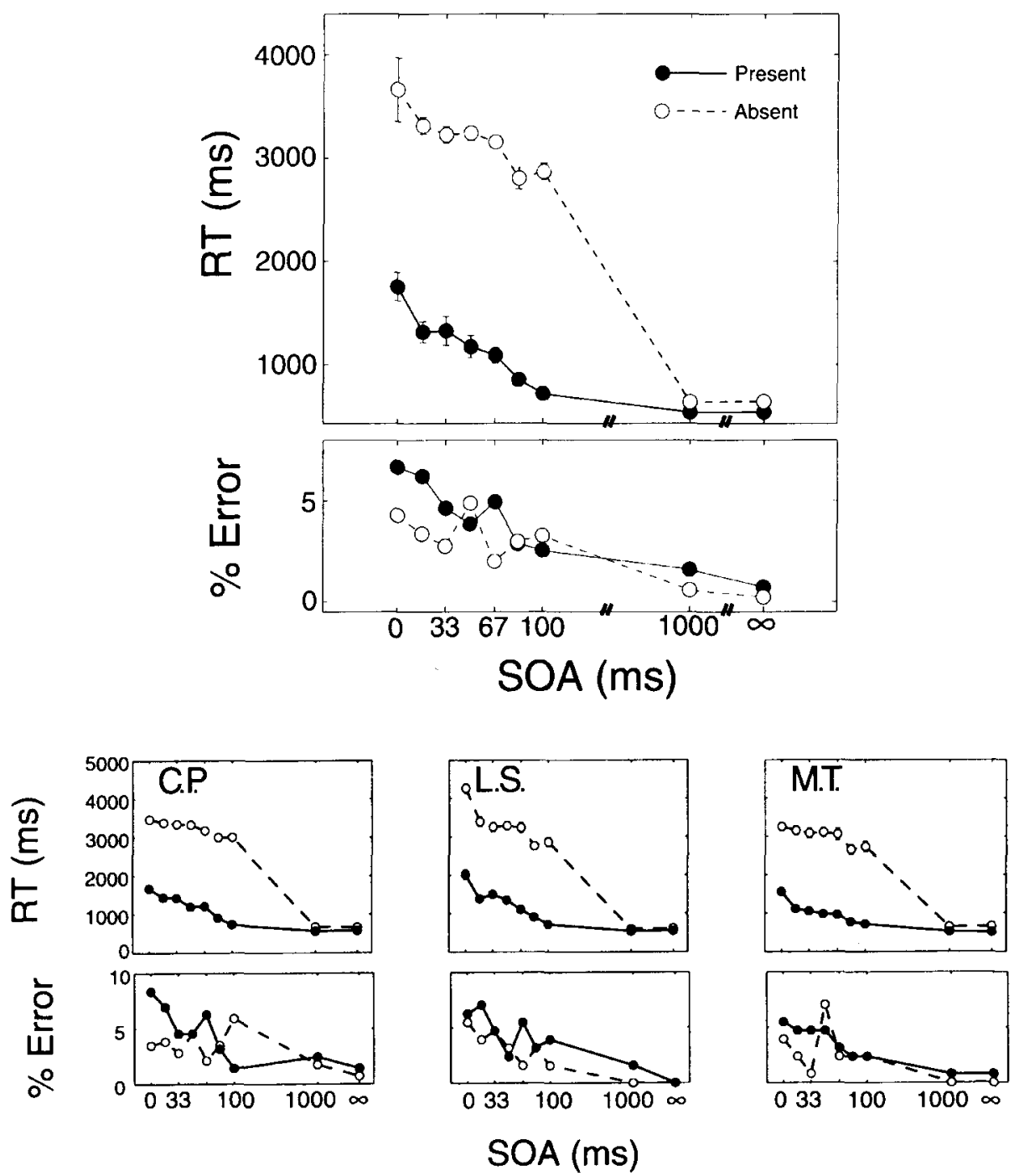

Figure 2. Mean reaction time (RT) and percent error for target-present (filled circles) and targetabsent (empty circles) trials, for trials in the different stimulus onset asynchrony (SOA) conditions, averaged across observers (top panel) and for individual observers (bottom panel). 
because target-absent trials are believed to involve a more varied range of strategies (Chun \& Wolfe, 1996). Mean RT tended to decrease with increasing SOA, until an SOA of $1,000 \mathrm{msec}$, where it was identical to that in the $\mathrm{SOA}=\infty$ condition. Pop-out was complete on all trials by $1,000 \mathrm{msec}$. After 1,000-msec exposure to the linearly separable display, interruption by D2 distractors did not interfere with search because the mechanisms responsible for pop-out search had already produced a detection response.

On some trials with shorter SOAs, the mechanisms responsible for pop-out were interrupted before they had a chance to produce a response (we know this because mean RT was longer for these conditions than for the $\mathrm{SOA}=\infty$ condition). In these cases, only the mechanisms responsible for difficult search could find the target, because pop-out could no longer operate once the D2 distractors appeared. It is these trials, where pop-out failed, that interest us. The conditions with shorter SOAs show the effect of incomplete pop-out on the difficult search that is necessary to find the target when pop-out has been interrupted. This effect, or interaction, is assessed using the RT distributions for the different SOAs. We characterize each RT distribution using its first four moments, where the $j^{\text {th }}$ moment, $\bar{x}^{j}$, is

$$
\overline{x^{j}}=\frac{\sum_{i=1}^{N}\left(x_{i}^{j}\right)}{N} .
$$

The first moment $\overline{x^{j}}$ is the sample mean. The second, third, and fourth moments $\left(\overline{x^{2}}, \overline{x^{3}}, \overline{x^{4}}\right)$ are related (but not identical) to the variance, skewness, and kurtosis, respectively. We describe the RT distributions in terms of their first four moments because these descriptors are sufficient to determine whether the intermediate RT distributions are linear combinations of the control RT distributions (difficult search in the SOA $=0$ control condition and pop-out search in the SOA $=\infty$ control condition), using a method described below.

We ask whether each intermediate distribution can be expressed as a convex combination (linear combination with nonnegative coefficients) of the two control distributions. If so, we can conclude that the mechanisms responsible for pop-out and difficult search are independent of each other and operate without interacting (as in race models and mixture models; see Olds et al., 2000b, for further discussion). Otherwise, we can conclude that there is some interaction (or overlap) between the processes responsible for pop-out and the processes responsible for difficult search.

To illustrate, imagine that pop-out search succeeds on $70 \%$ of the trials for a given intermediate SOA. If the independence hypothesis is true, then that intermediate RT distribution results from combining $70 \%$ pop-out search with $30 \%$ difficult search. ${ }^{3}$ It follows that the moments of this intermediate distribution will be in these same proportions as well. In other words, the first moment will be equal to $\left(0.7 * \bar{x}_{(\mathrm{SOA}=\infty)}+0.3 * \bar{x}_{(\mathrm{S}}(\mathrm{SA}=0)\right)$. Likewise, the second moment will be $\left(0.7 * \bar{x}^{2}{ }_{(\mathrm{SOA} \infty)}+0.3 *\right.$ $\bar{x}^{2}(\mathrm{SOA}=0)$, and so on.

We define a mixing parameter, $\lambda$, to be the proportion of trials where the difficult mechanism finds the target (under the independence hypothesis). We define $\lambda$ to be 1 when SOA $=0$, because pop-out does not operate in a display that is not linearly separable; $\lambda=0$ in the popout control condition $(\mathrm{SOA}=\infty)$. We expect that with increasing SOA (i.e., increasing exposure to the linearly separable display), pop-out will succeed on an increasing number of trials and $\lambda$ will decrease.

We can estimate $\lambda$, the proportion of difficult search, separately for each of the moments, for each of the intermediate distributions. By the independence hypothesis, each moment will be combined in the same proportion for any particular SOA (see Olds et al., 2000a, for algebraic demonstrations and Monte Carlo simulations demonstrating this prediction). Using each of the four moments separately, we then express each intermediate SOA (17$1,000 \mathrm{msec}$ ) as a weighted sum of the two control distributions ( $\mathrm{SOA}=0$ and $\mathrm{SOA}=\infty$ ), using the following equation:

$$
\begin{aligned}
\overline{x^{j}{ }_{(\mathrm{SOA})}}= & \lambda j_{(\mathrm{SOA})} * \overline{\left.x^{j}{ }_{(\mathrm{SOA}}=0\right)} \\
& +\left(1-\lambda j_{(\mathrm{SOA})}\right) * \overline{\left.x^{j}{ }_{(\mathrm{SOA}}=\infty\right)}
\end{aligned}
$$

That is, we define a mixing parameter $\lambda$ to be the proportion of difficult search evidenced by a given RT distribution [and $(1-\lambda)$ is the proportion of pop-out search].

If a given intermediate distribution is in fact a weighted sum of the two control distributions, then the same $\lambda$ (proportion difficult search) will be derived based on each of the four moments. If not, each moment will yield a different estimate of $\lambda$.

Figure 3 shows that the $\lambda$ s derived from the four moments do not coincide. Therefore, the intermediate distributions are not weighted linear sums of the control distributions.

In addition, with increasing SOA, the $\lambda$ s derived from higher moments fall faster than those derived from lower moments. Higher moments pertain to the tail of a distribution, so this consistent pattern indicates that the tail of the distribution shrinks with increasing SOA faster than the mean does (our distributions tend to be right-tailed, so this means that with increasing SOA, long RTs are speeded more than the decrease in mean would indicate, given linear combination). In other words, at intermediate SOAs something is assisting difficult search when pop-out fails.

It has been shown that targets are detected faster and more accurately when they appear close to fixation; this phenomenon has been termed the eccentricity effect (Carrasco, Evert, Chang, \& Katz, 1995; Carrasco \& Frieder, 1997; Wolfe, O'Neill, \& Bennett, 1998). Figure 4 shows mean RT and percent error for target-present trials as a function of SOA, plotted separately for targets appearing in each of four orbits (target positions located roughly 

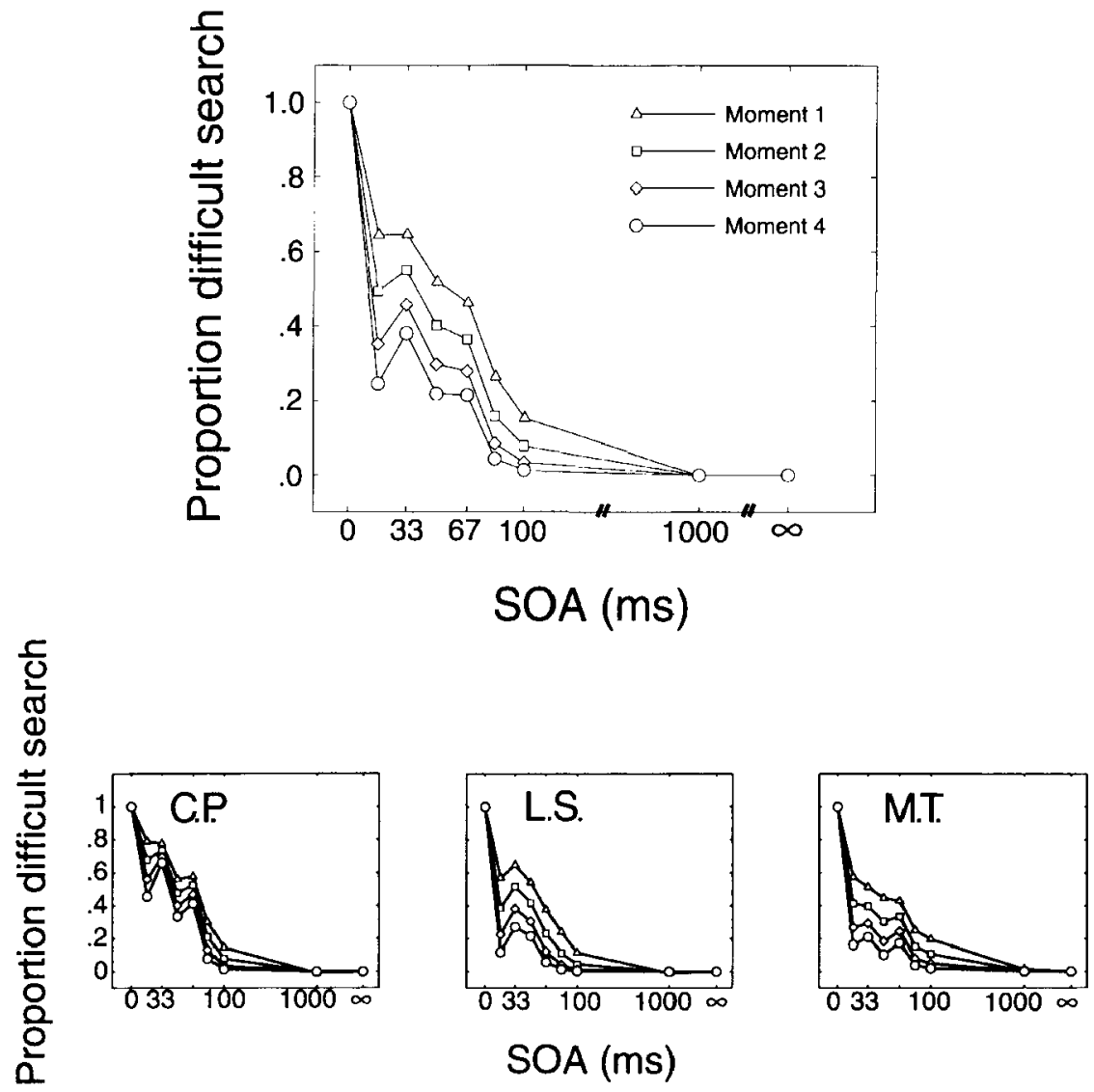

Figure 3. Proportion difficult search $(\lambda)$ derived from the first four moments of the hit reaction time (RT) distributions for each stimulus onset asynchrony (SOA) condition (top panel, mean of the individual observers' $\lambda$ s; bottom panel, individual observers' $\lambda s$ ).

$0.8^{\circ}, 1.9^{\circ}, 2.9^{\circ}$, and $3.4^{\circ}$ eccentricity from fixation). Targets in inner orbits tend to be detected before targets in outer orbits, and the errors follow the same pattern.

At $\mathrm{SOA}=0$, the advantage for inner orbits is simply a replication of the eccentricity effect for difficult search for color targets (although Orbits 1 and 2 are reversed for $\mathrm{RT}$, the ordering for errors by orbit is clear). At intermediate SOAs, the advantage of proximity to fixation is evident from the general trend of lower RTs and errors for targets nearer fixation than for those farther from fixation. This eccentricity effect for stimuli involving partial pop-out has not been shown before, except by Olds et al. $(2000 \mathrm{a}, 2000 \mathrm{~b})$ for color stimuli.

\section{DISCUSSION}

These data clearly show that partial pop-out helps difficult search in the orientation domain as well as in the color domain. The mixture analysis from the detection RT distributions demonstrates that when pop-out is interrupted before it has a chance to produce a detection response, its partial computations can be used by the mechanisms responsible for difficult search.
This generalization of the findings of Olds et al. (2000a, 2000b) demonstrates that the assistance of difficult search by partial pop-out is not limited to color. Our results indicate that this assistance may in fact be a general principle for visual selection.

Early visual selection determines which portions of the visual scene receive detailed analysis, so it is not surprising that it has an impact on all other visual perception. Investigating the interactions between different types of selection will deepen our understanding of how humans make sense of the visual world. Our technique is well suited to such investigations.

Furthermore, our technique provides a means for assessing the duration of the perceptual components of popout search, independent of overall RT (which includes motor and other nonperceptual components). The SOAs used in the present experiment do not allow us to definitively place an upper bound on this duration below $1,000 \mathrm{msec}$; however, it is clear from Figure 2 that an SOA of approximately $120 \mathrm{msec}$ might be expected to produce pop-out on all trials, given a linear extrapolation of mean RTs for SOAs of $0-100 \mathrm{msec}$. For color stimuli, Olds et al. $(2000 \mathrm{a}, 2000 \mathrm{~b})$ found the duration of the perceptual 

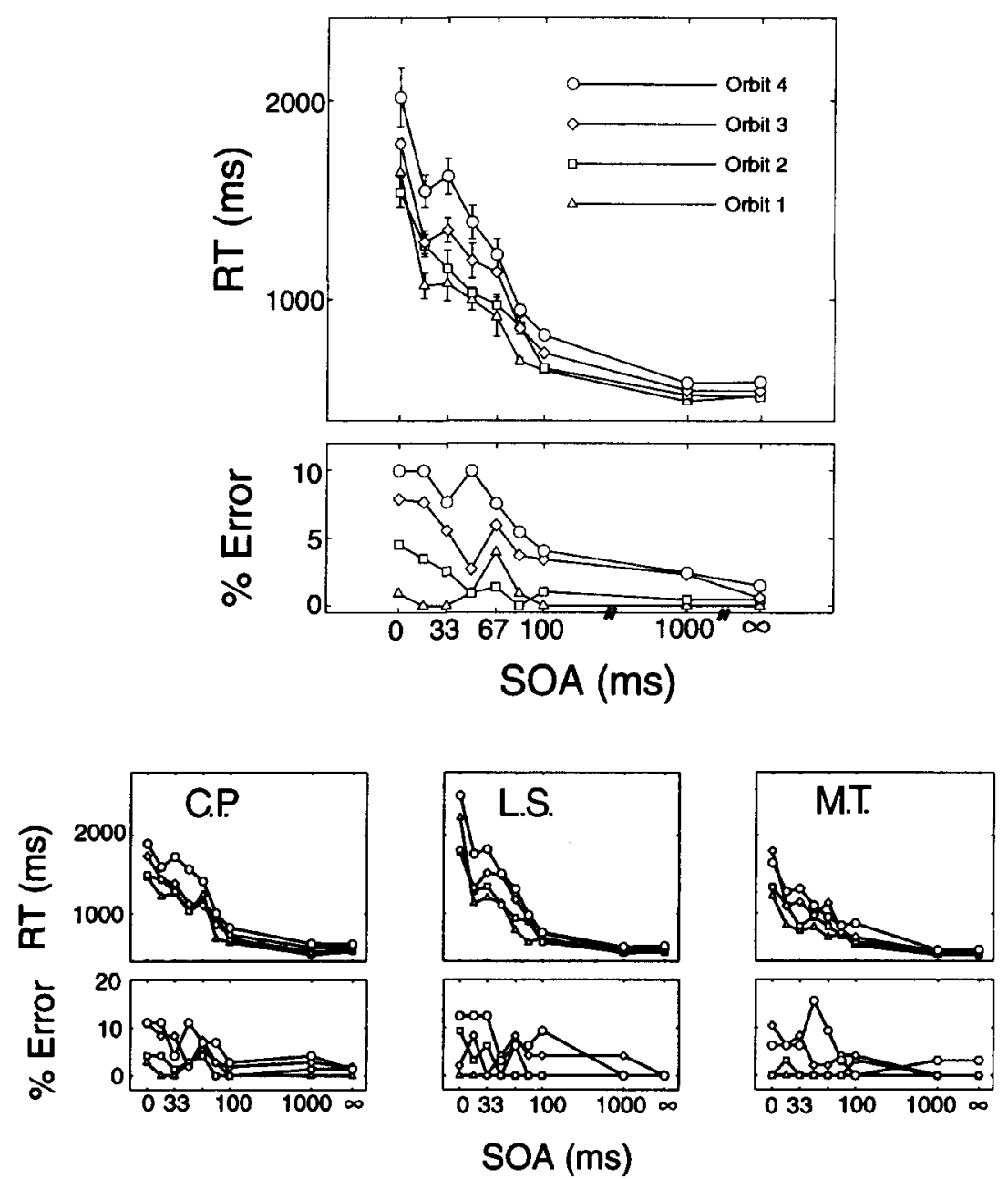

Figure 4. Mean reaction times (RTs) and percent error for target-present trials as a function of stimulus onset asynchrony (SOA), plotted separately for targets appearing in each of the four orbits. Please see text for further discussion.

components of color pop-out to be around $200 \mathrm{msec}$. This technique provides a useful way to measure and compare the perceptual components of pop-out processing for different kinds of stimuli.

Manipulation of discriminability would be expected to make the task more difficult. For example, a $45^{\circ}$ target would be easier to find among $45^{\circ} \pm 20^{\circ}$ distractors than among $45^{\circ} \pm 14^{\circ}$ distractors (the latter distractors were used in the present experiment). We would expect this decreased difficulty to result in a decrease in RT for search in both distractors (mean RT at SOA $=0$ ). Preliminary evidence from a conjunction search task (Jolicour, Olds, \& Cowan, 1999) indicates that this is the case. In addition, we would expect this difference in discriminability to affect the duration of the perceptual components of pop-out search. That is, the greater discriminability would also manifest itself as a decrease in the inflection point on the $x$-axis (this is the minimum SOA that produces pop-out performance; it is our measure of the duration of the perceptual components of pop-out search). Preliminary evidence (Jolicœur et al., 1999) indicates that this is the case as well. A manipulation of set size, on the other hand, might be expected to affect mean RT at SOA $=0$ (because set size affects difficult search RT) without changing the inflection point (duration of popout and pop-out RTs are only minimally affected by set size). This question has yet to be answered definitively, but preliminary results in our lab indicate that this is indeed the case.

What is the mechanism by which partial pop-out assists difficult search? Olds et al. (2000a) performed a set of experiments similar to the experiment described here, but with color stimuli. In almost all of these experiments, the same pattern resulted in the $\lambda$ s as is shown in Figure 3 . That is, we find the same pattern in the $\lambda$ s for both color stimuli and orientation stimuli. However, in a final experiment (Olds et al., 2000a, Experiment 7), the target changed location when the second set of distractors was 
added. That is, the target appeared in one location during the easy search portion of the trial, and then in a different location during the difficult search portion of the trial. The $\lambda$ s derived from the first four moments of the intermediate RT distributions coincide as a result of this manipulation and do not show the pattern of Figure 3. Coincident $\lambda$ s indicate that partial pop-out provides no assistance to difficult search, as discussed above. That is, when the target is in one location during partial pop-out processing and then in a different location for subsequent difficult search, the partial pop-out information cannot be used to speed the difficult search. Therefore, we can conclude that when partial pop-out does assist difficult search, it must do so via information about where the target is (or, alternately, where the target is not; see Olds et al., 2000a).

In the Guided Search model (Wolfe, 1994; Wolfe et al., 1989), basic feature differences make search easy by determining an optimal order for a difficult search process. What we call easy search occurs whenever basic feature differences allow difficult search consistently to process the target before any of the distractors. The results when the target is moved (Olds et al., 2000a) show that partial pop-out provides location information which can be used by difficult search processes. Thus, the information we see transferred for color in those experiments, and for orientation in the present experiment, can easily be the information transfer postulated by the Guided Search model.

Another set of results (Watson \& Humphreys, 1997) indicates another way that early processing can guide search. Watson and Humphreys's studies show evidence that locations known not to contain the target are not considered by difficult search processes (one could call this an excluded items hypothesis). Our results are also consistent with the guidance of difficult search by information about where the target is not (see Olds et al., $2000 \mathrm{a}$ ). As processing progresses and partial pop-out proceeds to completion, information about where the target $i s$, and information about where the target is not, will both increase. As a result, if pop-out is interrupted before completion, it will transmit to difficult search increasingly useful guidance, of whatever form.

For completed pop-out search there is an obvious relationship between information about where the target is (one single location) and where it is not (all other locations). This makes it very hard to discriminate experimentally between the Guided Search model and the excluded items model. Under conditions of partial pop-out, however, different heuristics are likely to describe the regions processed by the different models. Partially guided search, for example, is likely to be described by a series of contiguous regions that converge on the target over time; partial pop-out under excluded items assumptions, by contrast, may be described by early processing of targetfree clusters of distractors. More detailed development of the experimental paradigm described in this paper is the best way to discriminate and quantify the processing hypothesized by these two influential models.

\section{REFERENCES}

Bauer, B., Joliceur, P., \& Cowan, W. B. (1996), Visual search for colour targets that are or are not linearly-separable from distractors. Vision Research, 36, 1439-1466.

Bauer, B., Joliceur, P., \& Cowan, W. B. (1999). Convex hull test of the linear separability hypothesis in visual search. Vision Research, 39, 2681-2695.

BrainaRD, D. H. (1997). The psychophysics toolbox. Spatial Vision, 10, 433-436.

Carrasco, M., Evert, D. L., Chang, I., \& Katz, S. M. (1995). The eccentricity effect: Target eccentricity affects performance on conjunction searches. Perception \& Psychophysics, 57, 1241-1261.

Carrasco, M., \& Frieder, K. S. (1997). Cortical magnification neutralizes the eccentricity effect in visual search. Vision Research, 37, 63-82.

Chun, M. M., \& Wolfe, J. M. (1996). Just say no: How are visual searches terminated when there is no target present? Cognitive Psychology, 30, 39-78.

D'Zmura, M. (1991). Color in visual search. Vision Research, 31, 951-966.

Jolicceur, P., Olds, E. S., \& CowaN, W. B. (1999). Non-destructive interruption of pop-out search. Paper presented at the third annual Vision Research Conference, Fort Lauderdale, FL.

Olds, E. S., Cowan, W. B., \& Joliceur, P. (1999). Effective color CRT calibration techniques for perception research. Journal of the Optical Society of America A, 16, 1501-1505.

OldS, E. S., Cowan, W. B., \& Joliccur, P. (2000a). The time-course of pop-out search. Vision Research, 40, 891-912.

OldS, E. S., COWAN, W. B., \& Jolicceur, P. (2000b). Tracking visual search over space and time. Psychonomic Bulletin \& Review, 7, 292-300.

Treisman, A. M., \& Gelade, G. (1980). A feature-integration theory of attention. Cognitive Psychology, 12, 97-136.

VAN SELST, M., \& Joliceur, P. (1994). A solution to the effect of sample size on outlier elimination. Quarterly Journal of Experimental Psychology, 47A, 631-650.

Watson, D. G., \& Humphreys, G. W. (1997). Visual marking: Prioritizing selection for new objects by top-down attentional inhibition of old objects. Psychological Review, 104, 90-122.

WOLFE, J. M. (1994). Guided Search 2.0: A revised model of visual search. Psychonomic Bulletin \& Review, 1, 202-238.

Wolfe, J. M., Cave, C. R., \& Franzel, S. L. (1989). Guided search: An alternative to the feature integration model for visual search. Journal of Experimental Psychology: Human Perception \& Performance, $15,419-433$

Wolfe, J. M., Friedman-Hill, S. R., Stewart, M. I., \& O'Connell, K. M. (1992). The role of categorization in visual search for orientation. Journal of Experimental Psychology: Human Perception \& Performance, 18, 34-49.

Wolfe, J. M., O'Neill, P., \& BenNett, S. C. (1998). Why are there eccentricity effects in visual search? Visual and attentional hypotheses. Perception \& Psychophysics, 60, 140-156.

Wyszecki, G., \& STILES, W. S. (1982). Color science: Concepts and methods, quantitative data and formulae (2nd ed.). New York: Wiley.

\section{NOTES}

1. Control experiments have shown that the onset of extra distractors in itself only minimally disrupts pop-out search (Olds et al., 2000a).

2. Observer C.P. performed a session of warm-up trials, practicing only the SOA $=0$ and SOA $=\infty$ conditions, between the first four sessions and the last four sessions, because several weeks had elapsed between the two sets of sessions.

3. In a race model, the process to finish first provides the response. So technically this is the minimum of the two distributions. However, since the pop-out distribution is quite narrow relative to the difficult search distribution, and the two overlap minimally, this simplification suffices. See Olds et al. (2000) for further discussion.

(Manuscript received September 21, 1999; revision accepted for publication October 4, 1999.) 